\title{
El paisaje en el espacio público como medio de integración entre la ciudad y el agua: el caso de la región La Plata, Argentina
}

The landscape in the public space as an integration medium between the city and the water: The case of the La Plata region,

\section{Argentina}

\section{Mariana Birche* y Karina Jensen**}

Recibido: 30 de marzo de 2018

Aceptado: 05 de diciembre de 2018

\section{Resumen}

El presente artículo sostiene que la forma del crecimiento urbano actual junto con un entorno urbano fragmentado y la falta de propuestas sobre los espacios públicos significaría una pérdida en los valores de integración paisajística, fundamentalmente en los espacios públicos adyacentes a los cursos de agua. Se releva el área de estudio, con el caso de la región de La Plata, Argentina en pos de constatar la desvinculación existente entre los cursos de agua y área urbana residencial y, por ende, de uno de sus paisajes característicos. Para esto, se hace foco particularmente en aquellos espacios abiertos urbanos adyacentes a los cursos de agua, concebidos como elementos clave y potenciales disparadores para la mejora del hábitat. En este contexto, en el cual la ciudad tiende a la dispersión, cabe preguntarse cómo intervenir en estos espacios de manera que otorguen cualidad y calidad al ambiente construido. La metodología propone identificar las características fundamentales de la expansión urbana para comprender las lógicas de expansión y las principales formas y elementos que configuran el paisaje. A partir de esto, se asignan niveles de integración a los cursos de agua en todo su recorrido y finalmente desde la noción de paisaje, se propondrán estrategias para la creación de una red de agua y espacios verdes que respondan a las nuevas demandas de la población.

Palabras clave: ciudad intermedia, espacio público, integración urbana y paisajística, paisaje, ríos y arroyos urbanos.

\begin{abstract}
This article argues that the current form of urban growth together with a fragmented urban environment and the lack of proposals on public spaces would mean a loss in the values of landscape integration, mainly in the public spaces adjacent to the watercourses. The case study is La Plata region, Argentina, in order to verify the existing separation between the water courses and residential urban area and, therefore, one of its characteristic landscapes. For this, the research focuses particularly on those urban open spaces adjacent to watercourses, conceived as key elements and potential triggers for habitat improvement. In this context, in which the city tends to dispersion, it is worth asking how to intervene in these spaces in a way that improves the built environment. The methodology identifies the fundamental characteristics of urban expansion to understand the logics of expansion and the main forms and elements that make up the landscape. From this, levels of integration are assigned to the water courses throughout their journey. And finally, from the notion of landscape, it will be proposed strategies for the creation of a water network and green spaces that respond to the new demands of the population.

Keywords: intermediate city, landscape, public space, urban and landscape integration, urban rivers and streams.
\end{abstract}

\footnotetext{
* Filiación: Instituto de Investigaciones y Políticas del Ambiente Construido, Consejo Nacional de Investigaciones Científicas y Técnicas - Universidad Nacional de La Plata (IIPAC-CONICET/UNLP). Contacto: marianabirche@gmail.com

** Filiación: Instituto de Investigaciones y Políticas del Ambiente Construido, Consejo Nacional de Investigaciones Científicas y Técnicas - Universidad Nacional de La Plata (IIPAC-CONICET/UNLP). Contacto: karinacjensen@gmail.com
}

Cómo citar: Birche, M. y Jensen, K. (2018). El paisaje en el espacio público como herramienta de integración entre la ciudad y el agua: el caso de la región La Plata, Argentina. Revista de Urbanismo, 39, 1-16. https://doi.org/10.5354/0717$\underline{5051.2018 .48835}$ 


\section{Introducción}

En Latinoamérica, los límites entre lo rural y lo urbano se desdibujan debido a los procesos de dispersión y difusión urbana que tuvieron lugar en las últimas décadas, dando lugar a grandes áreas periurbanas de muy poca densidad (Herrera y Pecht, 1976; Lanfranchi, Duarte y Granero, 2018; ONU Habitat, 2012). De esta forma, como señalan Pascual y Peña (2012):

La ciudad compacta tradicional empieza a desvirtuarse frente a las presiones del proceso de urbanización. Por otro lado, la ciudad difusa consume áreas potenciales de cultivo, genera un incremento de las distancias e infraestructuras y un aumento de las demandas de transporte y energías. De esta manera, la zona urbanizada se extiende y se modifican los espacios naturales circundantes y sus ciclos biológicos, unido a un deterioro de la cohesión social (p.26).

En Argentina, a partir de finales del siglo XX, el Estado modifica sus acciones en pos de posibilitar $y$ facilitar grandes inversiones en la ciudad, de capitales nacionales y extranjeros. Para ello, se iniciaron procesos de desregulación político-administrativa del suelo urbano, que incrementaron la participación del mercado en la configuración de las ciudades (Clichevsky, 2001). Es así como las ciudades deben resistir en el largo plazo los efectos de la lógica cortoplacista, que por un lado suman el incremento de población, de espacio construido, de automóviles, de actividades y de estrés urbano. Y por otro, resta cantidad de espacios verdes públicos, de espacios colectivos, de espacios para los peatones, de asoleamiento, de superficies de suelo absorbente y de forestación, entre otros. La aplicación de políticas urbanas orientadas desde la priorización de la lógica económica arroja como resultante la pérdida de paisaje y de calidad de vida urbana.

Estas formas de expansión en la ciudad de La Plata en particular están configurando grandes áreas periféricas compuestas por espacios social y culturalmente heterogéneos, donde conviven tanto los sectores más pobres de la población como los de mayor nivel adquisitivo. Estos últimos, buscan una organización privada y eficiente de su vecindario que les provea de los servicios que antes solían ser públicos. En esta búsqueda, se han desplazado hacia las áreas periféricas de la ciudad para establecer su residencia en urbanizaciones cerradas, las cuales funcionan como guetos aislados del tejido urbano, propiciando la fragmentación del espacio urbano. De esta forma, en la ciudad latinoamericana en general y en las ciudades argentinas en particular el proceso de segregación socioespacial está siendo llevado a extremas consecuencias, colaborando con la configuración de una ciudad cada vez más fragmentada. Siguiendo esta línea, Dematteis (1998) relaciona la posición topográfica de la ciudad y su distinción en centro y periferia como una metáfora que esconde una marcada separación entre las valoraciones de ambas partes:

El criterio lateral de la posición topográfica (la periferia comprendida como parte de la ciudad que rodea al centro) se carga de significados valorativos cuando se convierte en metáfora de dominación (el centro que decide y controla) y de dependencia (la periferia que se estructura pasivamente en función del centro, alojando aquello que el centro rechaza). También el criterio residual, según el cual la periferia no es una verdadera ciudad, ni verdadero campo, no es meramente descriptivo sino valorativo, en tanto que sugiere la imagen de un área en la que tanto los valores asociados al hecho urbano, como aquellos propios de la cualidad medioambiental son mínimos. Una imagen negativa de este tipo queda explicitada en las definiciones de la periferia como no-centro, por consiguiente, como espacio carente de los valores de centralidad (Dematteis, 1998, p.1).

Así, se puede sostener que el término periferia, refiere por un lado a la herencia geométrica propia de la palabra periferia (la circunferencia externa), y por otro, es heredera de la teoría social de los años sesenta (Hiernaux y Lindón, 2004).

En la presente investigación se aborda en una primera instancia teórica la expansión urbana desde la noción de paisaje para luego, en una segunda instancia, proponer la intervención e integración urbana desde el paisaje en el área de estudio. Para esto se propone una metodología de abordaje para el caso de estudio y posteriormente se presentan los resultados, las medidas de integración y las conclusiones desarrolladas en el trabajo. 


\section{Expansión urbana y paisaje}

En este contexto, "la nueva ciudad de la dispersión está surgiendo de forma acrítica, más preocupada por las urgencias de lo inmediato que por comprender su propio sentido" (Arias, 2003 p.53). Consecuentemente, la metrópolis latinoamericana actual se desarrolla hacia una ciudad de islas cuyas formas de conformación provienen tanto del asentamiento insular de estructuras y funciones desde el momento de su construcción, como también del posterior aislamiento de espacios urbanos preexistentes mediante la construcción de rejas o muros. Las estructuras insulares de la ciudad latinoamericana se han convertido en elemento determinante de su configuración espacial.

Es importante señalar que el quid del asunto no sea tanto defender compacidad por encima del sprawl, o la posición contraria, sino encontrar un punto de equilibrio (masa crítica) que, según Rueda (1997), haga viable la provisión de transportes, equipamientos y servicios por un grado adecuado de proximidad entre usos y funciones urbanas y de equilibrio del tejido urbano (Sanabria y Ramírez, 2017). En este sentido, como señala Lanfranchi et al. (2018), la densidad de población óptima ronda los 90 habitantes por hectárea, mientras que en los grandes aglomerados urbanos de Argentina la superficie ocupada en 2016 aumentó a 619.283 hectáreas, con una población de 28.815.469 habitantes. Así, la densidad bruta se redujo a 46,5 hab/ha, implicando costos más altos tanto para el Estado como para la población.

A partir de aquí, valorizar los espacios cuyos atributos favorezcan la apropiación y el desarrollo del espacio urbano resulta clave para la reestructuración espacial y funcional de las áreas degradadas en la ciudad. En escenarios urbanos de crecientes conflictos tanto urbanos como sociales, el paisaje como categoría, aporta herramientas que permiten establecer criterios para zonificar el suelo no urbanizable y para la catalogación y conservación de los elementos estructurales del territorio que definen en mayor medida el carácter de un paisaje. Es así como el paisaje actúa como instrumento de dinamización y de mejora de la calidad del espacio urbano y también como una herramienta útil para orientar los futuros desarrollos urbanísticos.

En este contexto, y de la misma manera que han proliferado las urbanizaciones cerradas como una nueva forma de habitar la ciudad, se podría decir que en torno al espacio público urbano sucede algo similar. De esta forma, la aparición de los grandes centros comerciales y otros espacios pseudo públicos aparecen no sólo como un inocente cambio en el paisaje urbano, sino, además, como un factor importante en la transformación de nuestras interacciones sociales y la tradición liberal moderna sobre cómo interpretar dicha interacción (Salcedo, 2002). La ciudad es un territorio protegido y protector que formalmente hace iguales a sus ciudadanos, pero las realidades físicas y sociales expresan a su vez la exclusión y el desamparo de unos frente a los privilegios y al pleno disfrute de las libertades urbanas de otros (Borja, 2003). Es por eso que:

La red de espacios públicos resulta clave para la ciudad. Esta red, permite la conexión de un punto a otro de la ciudad, la comunicación espontánea entre los ciudadanos y la percepción de lo urbano. La ciudad como hecho colectivo se manifiesta fundamentalmente en su red de espacios públicos. Estos espacios urbanos deben ser analizados como una red, pues consiste en un espacio continuo formado por diferentes elementos conectados entre sí. Dentro de las funciones que desempeñan se distinguen las siguientes: establecen una relación directa entre el espacio urbano y el territorio que lo rodea, constituyen el conjunto de canales de comunicación entre las diferentes partes de la ciudad, son la referencia permanente de la parcelación y, por último, focalizan y articulan el tejido urbano, aportándoles significado e imágenes reconocibles a las partes de la ciudad (Pascual y Peña, 2012 p.30).

En este sentido, las nuevas formas de entender el urbanismo y el planeamiento urbano desde la noción de paisaje surgen como reacción a los modelos de zonificación y urbanización en los que el potencial de un lugar se consume contraponiendo el lleno al vacío, la ciudad a la naturaleza, lo ordenado a lo conservado. En definitiva, un urbanismo en el que el paisaje se convierte además de herramienta de análisis, en instrumento creativo y en proyecto de lugar de la mano de la ecología y el diseño, generando una disciplina propia (García, 2015).

De esta forma, si bien desde el surgimiento del urbanismo como disciplina el paisaje se incorpora al 
diseño de las ciudades, desde mediados de los años sesenta del siglo pasado algunos sectores de la intelectualidad europea empiezan a mostrar inquietud por la preservación de los valores de los paisajes como un tema a ser estudiado en profundidad (UNESCO aprueba la Recomendación relativa a la protección de la belleza y el carácter de los lugares en 1962). Como resultado se observa la aparición de leyes y normas nacionales y regionales enmarcadas en políticas territoriales. ${ }^{1}$

A partir de esto, se puede sostener que existe una tendencia a la evolución desde una intención preferentemente estética y conservacionista, al protagonismo del desarrollo territorial. Ello supone un reforzamiento de las facetas económicas y sociales de los paisajes, entendidos éstos como marcos vitales, espacios para el disfrute ciudadano y activos para el desarrollo (Silva y Fernández, 2015).

De esta forma, el paisaje como disciplina debe constituirse como una proyección de lo contemporáneo, una disciplina que, desde la integración de nuevas perspectivas, brinde la oportunidad de repensarla en nuestro contexto social y cultural, con un objetivo doblemente arquitectónico y paisajístico. Esto es desde la perspectiva de crear un puente o un lugar de encuentro entre dos disciplinas que hoy pueden entenderse como concurrentes, precisamente porque los grandes trabajos que la sociedad demanda empiezan a exigir una formación integrada.

\section{El área de estudio}

El área de estudio o región del Gran La Plata constituye un aglomerado urbano alrededor de la ciudad de La Plata, capital de la Provincia de Buenos Aires, Argentina. El Gran La Plata está compuesto por los partidos de La Plata, Ensenada y Berisso y es la sexta aglomeración más poblada del país. Se encuentra al noreste de la provincia de Buenos Aires y muy cerca del límite sudeste del Gran Buenos Aires. Mientras que Ensenada y Berisso ocupan franjas costeras sobre el Río de la Plata, el partido de La Plata se extiende al sur de ellos y llega en un pequeño sector a ocupar tierras costeras, exactamente entre medio de los otros dos partidos.

En la región pampeana, las cuencas hidrográficas representan uno de los accidentes geográficos más relevantes. Su gestión ha significado históricamente un desafío en materia ambiental, por estar asociadas a graves niveles de contaminación e inundaciones urbanas, ya que la escasa pendiente hacia el río que poseen dificulta el correcto escurrimiento de las aguas frente a lluvias importantes. Sin embargo, persisten en torno a ellas valores ambientales y paisajísticos poco reconocidos, por lo cual este territorio se convierte en un lugar propicio para analizar la aplicación de herramientas que asocien planificación territorial y paisaje, contribuyendo a una mayor integralidad en los instrumentos de planificación actuales (Rotger, 2017).

El área de estudio es atravesada por seis cuencas que integran el sistema hídrico regional de la vertiente del Río de la Plata, las cuales en orden de aparición partiendo orientación de noroeste a sudeste, se encuentran la cuenca de arroyo Carnaval que comprende los arroyos Carnaval y Martín, la cuenca del arroyo Rodríguez que comprende el arroyo Rodríguez y el arroyo Don Carlos, la cuenca del arroyo del Gato a la cual pertenecen el arroyo del Gato como cauce principal y los arroyos Pérez y El Regimiento que se encuentran entubados bajo el casco fundacional. La de menor extensión es la cuenca del arroyo Jardín zoológico que encuentra al interior del tejido urbano, al Sureste del casco fundacional está presente la cuenca del arroyo Maldonado y por último se encuentra la cuenca del arroyo El Pescado.

Los arroyos de la región se pueden clasificar de según su ubicación (Figura 1):

- Arroyos del Norte: Carnaval, Martín, Rodríguez, Don Carlos Y Del Gato.

- Arroyos dentro del Casco Urbano Fundacional: Pérez, Regimiento Y Jardín Zoológico.

- Arroyos del Sur: Circunvalación, Maldonado, Garibaldi Y El Pescado.

\footnotetext{
${ }^{1}$ Como por ejemplo, la Ley Federal sobre la protección de la naturaleza y el paisaje suizo, 1966; la Ley Federal de protección de la naturaleza y
}

gestión del paisaje alemana, 1976; o la ley de protección y puesta en valor de los paisajes franceses, 1993. 


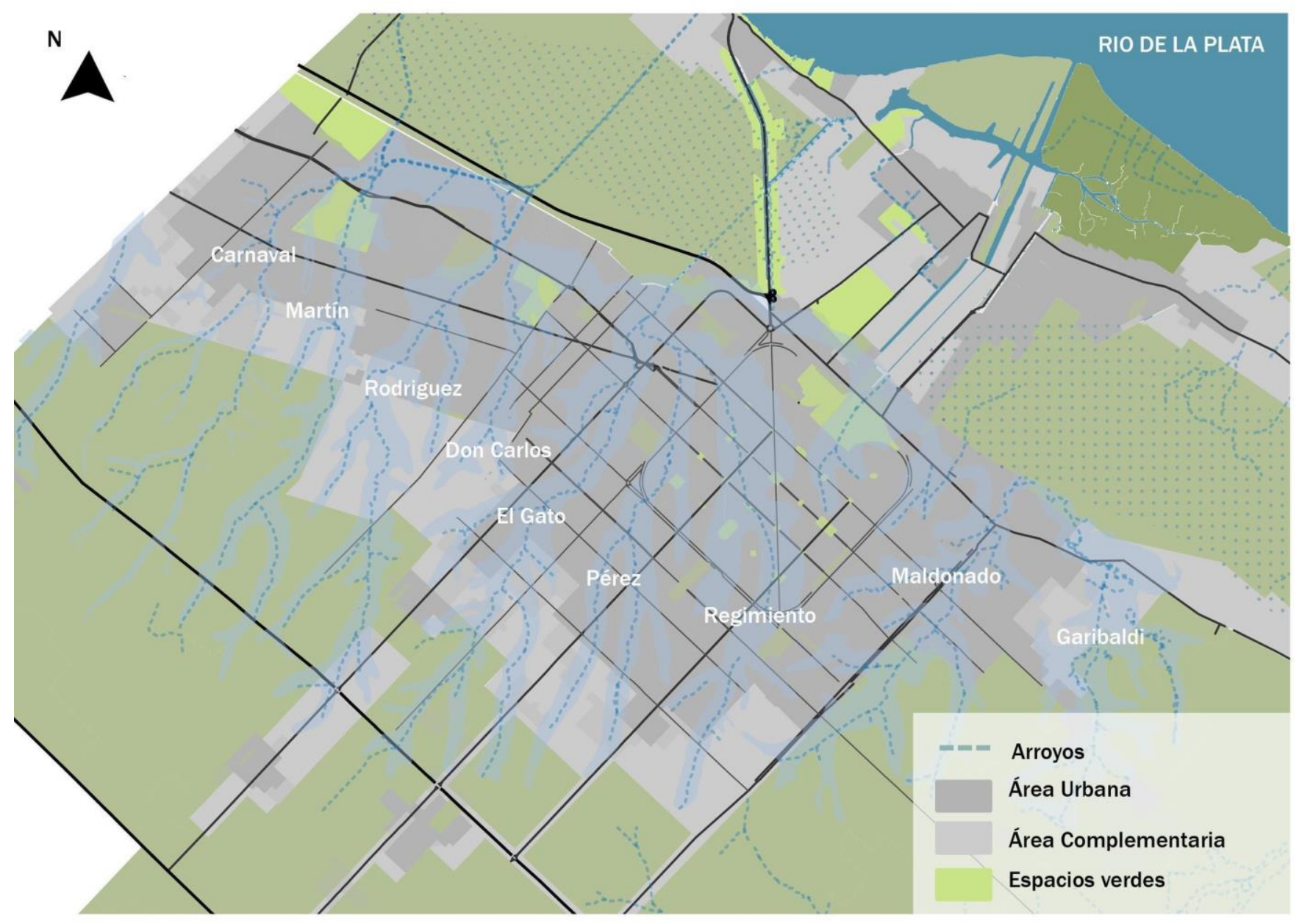

Figura 1. Los arroyos de la región.

Fuente: Elaboración propia, 2017.

En general se trata de pequeños cauces de poca profundidad, con canales de estiaje de unos pocos metros de ancho, con agua permanente sólo en la cuenca media y baja. La mayoría de los arroyos y cuerpos de agua presentan modificaciones debido a la actividad humana, una baja calidad de sus aguas ya que se encuentran afectados por los efluentes de las diversas actividades industriales (Romanazzi y Urbiztondo, 2011).
Los principales vertidos de residuos industriales se refieren a cobre, mercurio y plomo, hidrocarburos alifáticos y aromáticos, fenoles; grasas y aceites benceno, naftalenos, antracenos y toluenos (Jensen y Birche, 2017b); que mayoritariamente son descargados en forma directa en los cursos de agua, sobre todo en los canales Este y Oeste que circundan el polo petroquímico y en el recientemente canalizado arroyo El Gato (Figura 2). 

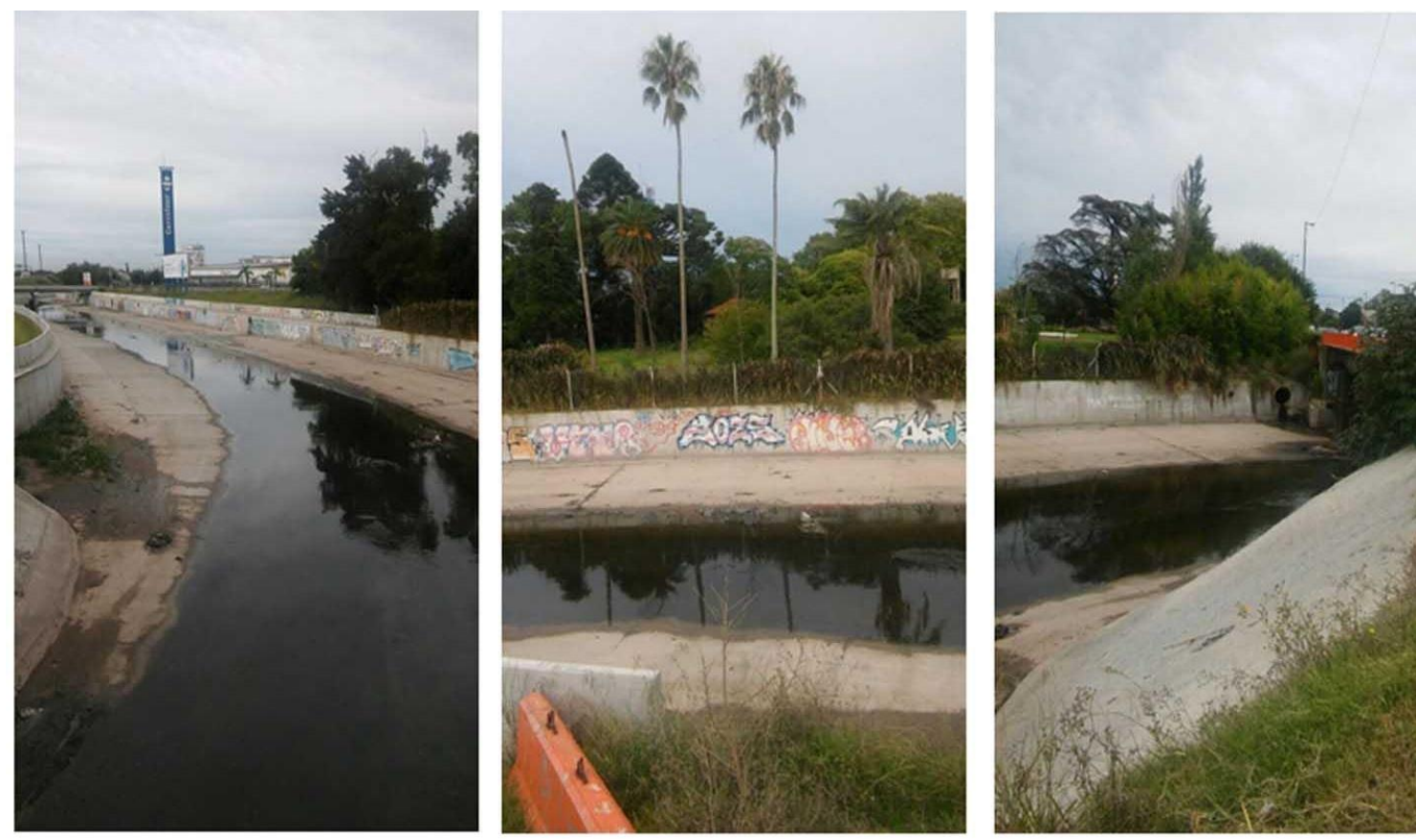

Figura 2. Nueva canalización del Arroyo El Gato.

Fuente: Archivo propio, 2017.

\section{EI paisaje como herramienta de intervención e integración urbana}

La expansión del área urbana en la región La Plata se produjo de manera vertiginosa principalmente en los últimos veinticinco años. Este fenómeno, impulsado (entre otras cosas) por el mercado inmobiliario y la falta de planificación, sumado a la imposibilidad de acceso al suelo urbano, produce una expulsión de la población hacia terrenos más alejados del centro (suelo productivo), lo que favorece la expansión de las periferias y la consolidación de los barrios más alejados. Este crecimiento hacia los terrenos productivos de la ciudad, configurando espacios urbanos fragmentados, que evidencian una baja calidad urbana y paisajística en estas nuevas zonas periféricas. Esta situación, en adición a la falta de planificación y la carencia de espacios verdes públicos genera un fuerte contraste con la situación dentro del casco urbano (Jensen y Birche, 2017a).

En la década de 1990, tuvieron lugar distintos procesos que dinamizaron la configuración urbana de ciudad de La Plata, particularmente el eje noroeste. Entre estos procesos, la inauguración de la Autopista La Plata - Buenos Aires (1996) y la readecuación de la Autovía 2 (1993-99), han favorecido la aparición de nuevos emprendimientos como barrios cerrados que propician la fragmentación socioespacial de la ciudad mientras que el espacio público se desvanece como ámbito común, general y colectivo con lo cual entran en crisis tanto la ética ciudadana de republicanismo de cuño liberal como la praxis social de raíz materialista (Arroyo, 2017).

Como sostiene Ábalos (2005, p.42), el paisaje es entendido como:

El efecto de la superposición de la actividad humana sobre la naturaleza, e incluye las modificaciones derivadas de la actuación sobre el medio para hacerlo productivo - parcelaciones, áreas cultivadas, minería, forestación y deforestación, dehesas, pastos y ganaderías, etc.- y construir artificialmente sobre él, sean infraestructuras -puentes presas caminos, etc.- o realizaciones propiamente arquitectónicas. Se trata, por tanto, de un término que implica una orientación proyectual clara y una condición híbrida, natural y artificial: la proyección de la cultura- tanto en su significado etimológico como el convencional- sobre el territorio natural.

Es decir que se puede entender el paisaje como la cultura territorial de una sociedad determinada, como el 
resultado de la interacción entre el hombre y la naturaleza. Este planteamiento tiene dos significados principales. El primero, es el reconocimiento de que el territorio contiene y expresa a través de la noción de paisaje, las formas de actuar del grupo humano que lo ocupa y lo maneja con distintos fines (de subsistencia, productivos, simbólicos). Y, por otra parte, reconoce que existen valores colectivos en las formas y en la organización del espacio vivido y que estos se asocian a la cultura propia, en tanto que proyecto o proyección futura de esa cultura.

Intervenir el territorio desde una mirada paisajística implica ser consciente de las transformaciones que en él se han dado y la importancia de pensar en las generaciones futuras. Implica también, reconocer la estrecha relación entre cultura y naturaleza, donde lo más importante es construir nuevos vínculos entre el hombre y el medio que lo rodea, en pos de establecer una relación armónica con el ambiente natural y reducir la vulnerabilidad frente a los fenómenos críticos que afectan la región. Por lo tanto, pensar nuevas estrategias desde la mirada del paisaje es pensar en una manera integral de ver y actuar en el territorio, teniendo en cuenta no sólo la dimensión física ambiental, sino también la dimensión social, cultural y estética con el fin de mejorar la calidad de vida de la población (Celemín, Mikkelsen y Velázquez, 2015).

Es así como se entiende al concepto de paisaje continuo como una oportunidad de integrar la ciudad (Pérez y Salinas, 2015). Hablar de una visión integral que permita ver más allá, es mirar las interrelaciones que se dan entre los sistemas y entender el problema desde un punto de vista reflexivo. Esto plantea una nueva forma de aproximación en la ciudad que permite actuar sobre ella, pero entendiendo que está llamado a formar parte de un ecosistema en constante transformación. El desafío más importante consiste en planificar coordinadamente tiempos diferenciados como lo son el tiempo humano y el tiempo natural, pensando siempre en la idea de totalidad. Siguiendo esta línea, la Real Academia Española (2017) define integrar como:

constituir un todo; completar un todo con las partes que faltaban; hacer que alguien o algo pase a formar parte de un todo; comprender; aunar, fusionar dos o más conceptos, corrientes, divergentes entre sí, en una sola que las sintetice.
De esta forma, según el Departamento de Medioambiente, Planificación Territorial y Vivienda del Gobierno Vasco (2016) se puede definir integración paisajística como aquella serie de acciones que conforman el proyecto y que están encaminadas a la implantación, ejecución y gestión de este, considerándolo con el lugar de manera armónica. Se puede decir entonces que Integración en el paisaje es la cualidad que tiene un proyecto o un espacio de formar parte de un paisaje, considerado en todos sus aspectos y facetas. La integración, por tanto, consiste en considerar el proyecto como un elemento que pasa a formar parte de los procesos ambientales, que constituyen la materia prima del paisaje, de la ocupación y utilización humana que convierte un espacio geográfico en territorio y de la estructura escénica y visual del paisaje, que lo convierte en cultura. Se considera una buena integración de un proyecto en el paisaje aquélla que establece, mantiene y consolida las facetas ambientales, territoriales, escénicas y estéticas del paisaje.

El concepto de paisaje ha entrado con toda legitimidad en el urbanismo, no como un complemento sino como un elemento principal, a veces incluso ordenador (Borja, 2003). Es así como el desafío del paisaje en la actualidad consiste en transformarse en un factor que se puede cualificar de las periferias difusas, de la ciudad en su conjunto y sobre todo de los nuevos espacios que aún resultan insignificantes.

\section{Metodología}

La presente investigación pretende verificar que el modelo de crecimiento actual juntamente con la falta de propuestas sobre los espacios públicos conformaría un entorno urbano fragmentado significando una pérdida de la calidad paisajística. Para esto, la metodología propone en una primera instancia identificar las características fundamentales de la expansión urbana en la región mediante un relevamiento exhaustivo del tejido que permita comprender las lógicas de expansión y las principales formas y elementos que configuran el paisaje. En una segunda instancia, se asignarán niveles de integración a los cursos de agua en todo su recorrido y, finalmente, en una tercera instancia, se proponen lineamientos y estrategias de intervención sobre una de las zonas de análisis trabajadas. 
En la primera instancia de trabajo, para abordar el relevamiento, se comenzó por identificar las distintas zonas que se conforman en relación a la estructura urbana y a la disposición de los principales usos de suelo. Se decide adoptar cuatro zonas de análisis, respetando las divisiones por dentro del partido (dieciocho centros comunales que constituyen delegaciones además del "Casco Urbano") $)^{2}$ y las tres zonas propuestas por el Código de Espacio Público de La Plata (Municipalidad de La Plata, 2004) en su Art. 10, pero subdividiendo la tercera zona que comprende el eje Noroeste y las localidades rurales del cinturón verde platense, entendiendo que responden a realidades y procesos de formación diferentes. Esta subdivisión, se corresponde con los principales ejes de crecimiento de nuestra ciudad (hacia Buenos Aires, hacia AU2 y hacia Ruta 11) y representa a su vez una agrupación de las delegaciones según problemáticas comunes. De esta forma, se adoptan las siguientes zonas (Figura 2):
- El casco urbano, delimitado por la Av. Circunvalación de acuerdo al diseño establecido desde su fundación.

- La zona de desborde, generada a partir de los primeros procesos de crecimiento por fuera del casco fundacional, se compone por las delegaciones adyacentes al casco urbano: Tolosa, San Carlos, Los Hornos, Altos de San Lorenzo y Villa Elvira.

- El eje de crecimiento NO, entendido a partir del crecimiento en torno a las principales vías de conectividad de la ciudad con la ciudad de Buenos Aires. Incluye las localidades de Villa Elisa, City Bell, Gonnet, Ringuelet, Gorina y Hernández.

- El cinturón verde se conforma por la Reserva Urbana del Área Complementaria y por el sector de la Zona Rural Intensiva. Se conforma a partir de las localidades de Arturo Seguí, Melchor Romero, El Peligro, Abasto, Etcheverry y L. Olmos y su crecimiento hacia la AU2.

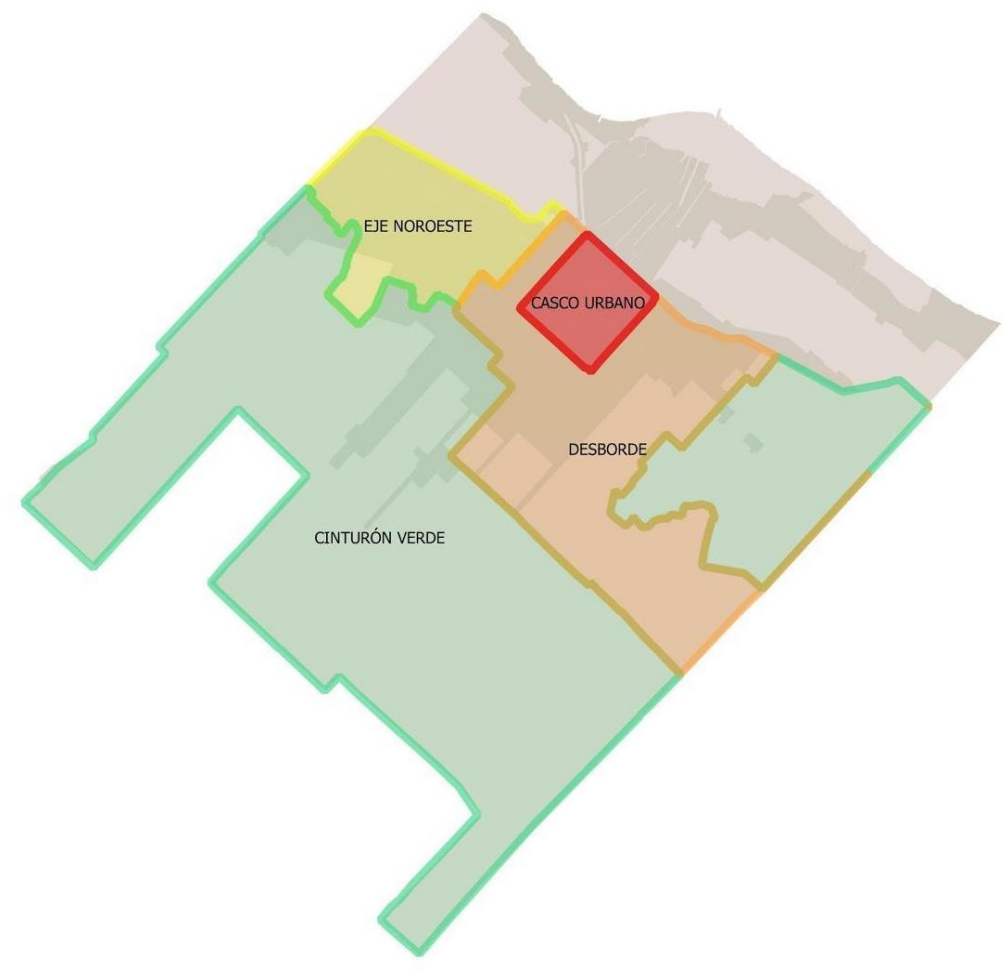

Figura 3. Zonas de análisis.

Fuente: Elaboración propia, 2017.

\footnotetext{
${ }^{2}$ Las delegaciones que conforman el Partido de La Plata terminaron de conformarse como tales en el año 1999 durante el mandato del intendente Julio Alak, ya que anteriormente estaban constituidas como
}

tales sólo siete de ellas (Villa Elisa, City Bell, Gonnet, Los Hornos, Villa Elvira, Tolosa y Romero) 
Tabla 1. Proyección de la población para el Partido de La Plata según zonas de análisis.

\begin{tabular}{cccccc}
\hline $\begin{array}{c}\text { Zonas de } \\
\text { análisis }\end{array}$ & $\begin{array}{c}\text { Población } \\
\text { Censo 1991 } \\
\text { Habitantes }\end{array}$ & $\begin{array}{c}\text { Población } \\
\text { Censo 2001 } \\
\text { Habitantes }\end{array}$ & $\begin{array}{c}\text { Población } \\
\text { Censo 2010 } \\
\text { Habitantes }\end{array}$ & $\begin{array}{c}\text { Población } \\
\text { estimada 2017 } \\
\text { Habitantes }\end{array}$ & $\begin{array}{c}\text { Crecimiento } \\
1991-2017 \\
\text { (\% Habitantes) }\end{array}$ \\
\hline Casco & $\mathbf{2 0 7 , 4 3 4 . 0}$ & $181,402.0$ & $191,075.0$ & $211,744.9$ & 102.1 \\
\hline Desborde & $202,294.0$ & $220,156.0$ & $228,538.0$ & $253,259.0$ & 125.2 \\
\hline Eje Noroeste & $\mathbf{6 2 , 6 4 8 . 0}$ & $\mathbf{1 0 4 , 7 5 1 . 0}$ & $\mathbf{1 5 9 , 7 8 3 . 0}$ & $\mathbf{1 7 7 , 0 6 6 . 3}$ & $\mathbf{2 8 2 . 6}$ \\
\hline $\begin{array}{c}\text { Cinturón } \\
\text { Verde }\end{array}$ & $69,529.0$ & $\mathbf{7 0 , 6 1 4 . 0}$ & $\mathbf{8 0 , 9 3 9 . 0}$ & $\mathbf{8 9 , 6 9 1 . 4}$ & 129.0 \\
\hline TOTAL LP & $\mathbf{5 4 1 , 9 0 5}$ & $\mathbf{5 7 4 , 2 2 3}$ & $\mathbf{6 6 0 , 3 3 5}$ & $\mathbf{7 3 1 , 7 8 3}$ & $\mathbf{3 5 . 0}$ \\
\hline
\end{tabular}

Fuente: Elaboración propia, 2017.

A partir de estas premisas, se elaboró el siguiente plano (Figura 3) que contiene los principales usos del suelo relevados y la proyección de aumento poblacional para el año 2017. Esta proyección, se elaboró a partir de establecer una tasa de crecimiento anual de 1,55\%, basada en el crecimiento intercensal entre el año 2001 y el 2010, lo que arroja un crecimiento de 71.448 habitantes que se distribuyen por zonas de análisis. En la Tabla 1, se puede apreciar, en conjunto con la Figura 4, por qué de las cuatro zonas de la periferia determinadas el trabajo hace foco en el eje noroeste, dado que resulta en la zona que presenta mayor grado de complejidad en relación con los usos y las dinámicas urbanas y a su vez es la que mayor porcentaje de crecimiento ha tenido en los últimos años casi triplicando su población.

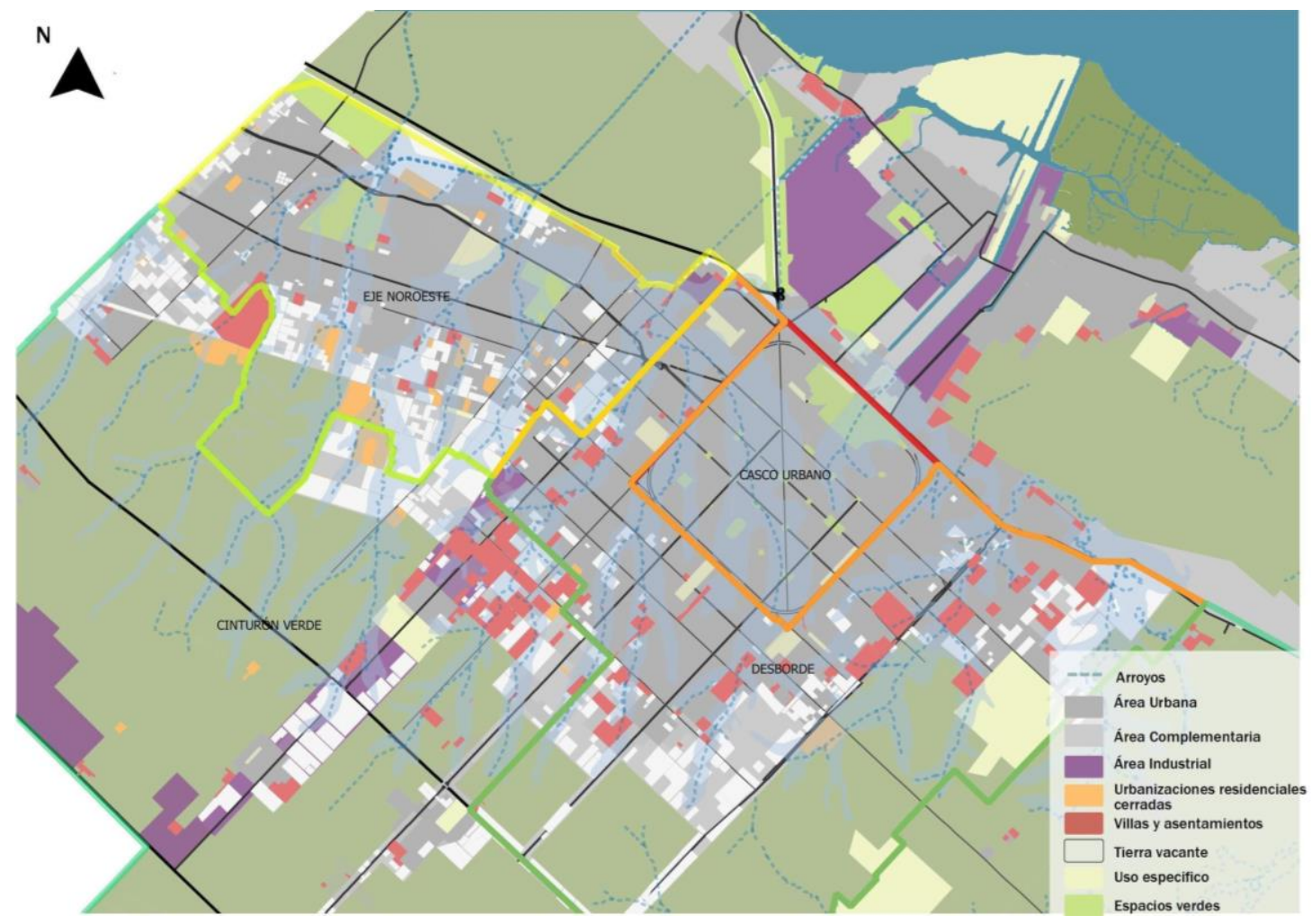

Figura 3. Cursos de agua y principales usos del suelo asociados para el Partido de La Plata.

Fuente: Elaboración propia. 2017 
A partir de este punto, el trabajo propone la asignación de tres niveles de integración en relación con las formas que evidencian y manifiestan la relación agua-ciudad. Se propone una división en bueno, regular y malo según los siguientes criterios:
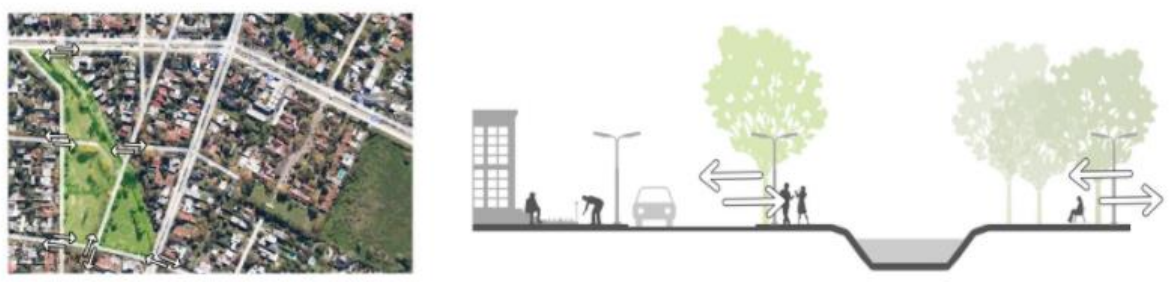

Figura 4: Nivel de integración Bueno.

Fuente: Elaboración propia. 2017

Nivel de integración paisajística bueno (Figura 4): La integración agua-ciudad se da mediada por espacios de construcción y apropiación social, en donde predominan la vegetación y los usos recreativos y de esparcimiento y también la contemplación del paisaje en cuencas visuales más amplias que las asociadas a la trama urbana regular.
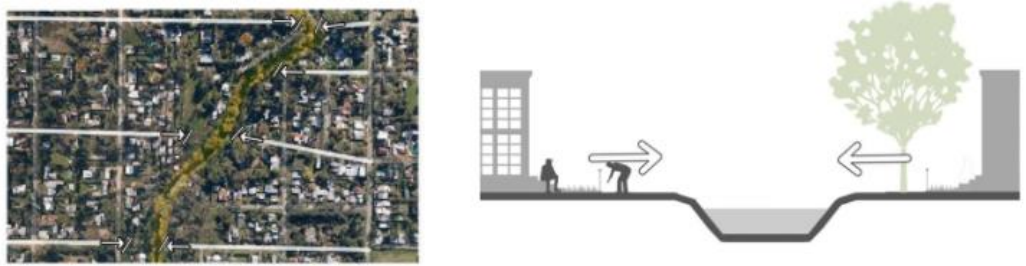

Figura 5. Nivel de integración Regular.

Fuente: Elaboración propia. 2017

Nivel de integración paisajística regular (Figura 5): La integración agua-ciudad se basa en la integración directa de la trama urbana los espacios asociados al borde inmediato del arroyo. Son espacios acotados y con escasa vinculación con el curso de agua
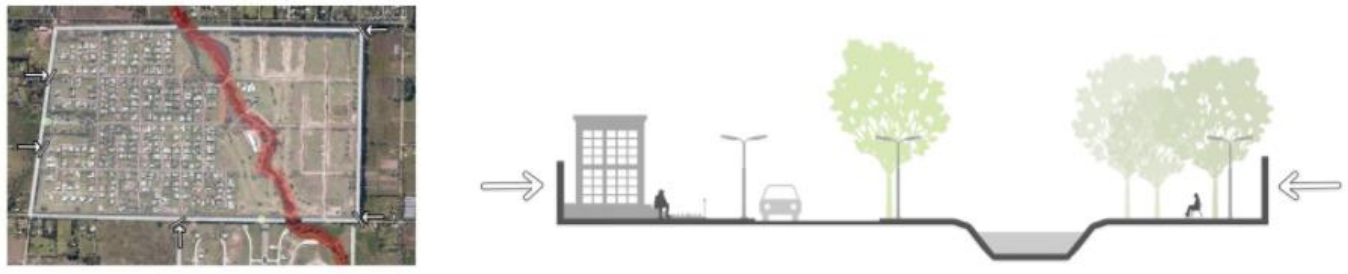

Figura 6. Nivel de integración Malo.

Fuente: Elaboración propia. 2017

Nivel de integración paisajística malo (Figura 6): La integración agua-ciudad se ve impedida por constituirse por dentro de un elemento urbano diferenciado, fragmentado del entorno. La relación con la ciudad es escasa o nula. 
El trabajo muestra los resultados de un proceso de investigación que se viene llevando a cabo en la región, que abarca principalmente el período de las tres últimas décadas. Forma parte de una investigación más amplia en base al proyecto de investigación acreditado por la UNLP para el período 2014-2018 “El paisaje como factor de mitigación de la degradación socio ambiental en la región La Plata" (Birche y Jensen, 2016) que aborda la problemática de los cursos de agua y los espacios abiertos en el planeamiento y el diseño urbano contemporáneo.

\section{Resultados}

En la zona de análisis que corresponde con el eje noroeste en particular se observan numerosos y diferentes usos en relación con los cursos de agua. Estos usos, determinan cómo se vinculan a los espacios del agua la trama urbana, el tejido residencial, los espacios verdes, los barrios semicerrados y urbanizaciones cerradas. En el plano se relevaron las diferentes situaciones y se marcaron con diferentes colores asociados al nivel de integración. Para comprender la situación planteada anteriormente como un problema de carácter espacial y poder determinar el nivel de relación entre el agua y la ciudad según los criterios de la integración paisajística, se realizó una cartografía en GIS a partir del relevamiento realizado a partir de observación indirecta a través de Google Earth y Google Street View y en una segunda instancia a través de trabajo de campo.

En la Figura 7, los tramos de los cursos de agua que poseen una buena integración paisajística aparecen en verde, los que poseen una integración regular en amarillo y los que poseen mala integración en rojo. De esta manera, se detectaron zonas que poseen buena integración y donde la ciudad vive esa relación aguaciudad de una manera fluida y también zonas donde el crecimiento urbano se da mediante urbanizaciones semicerradas o cerradas y la relación de la ciudad con los cursos de agua es nula. En el caso de los barrios y urbanizaciones cerradas particularmente se observa una buena integración en el interior, pero la relación de estos con la ciudad propiamente dicha se encuentra prácticamente anulada.

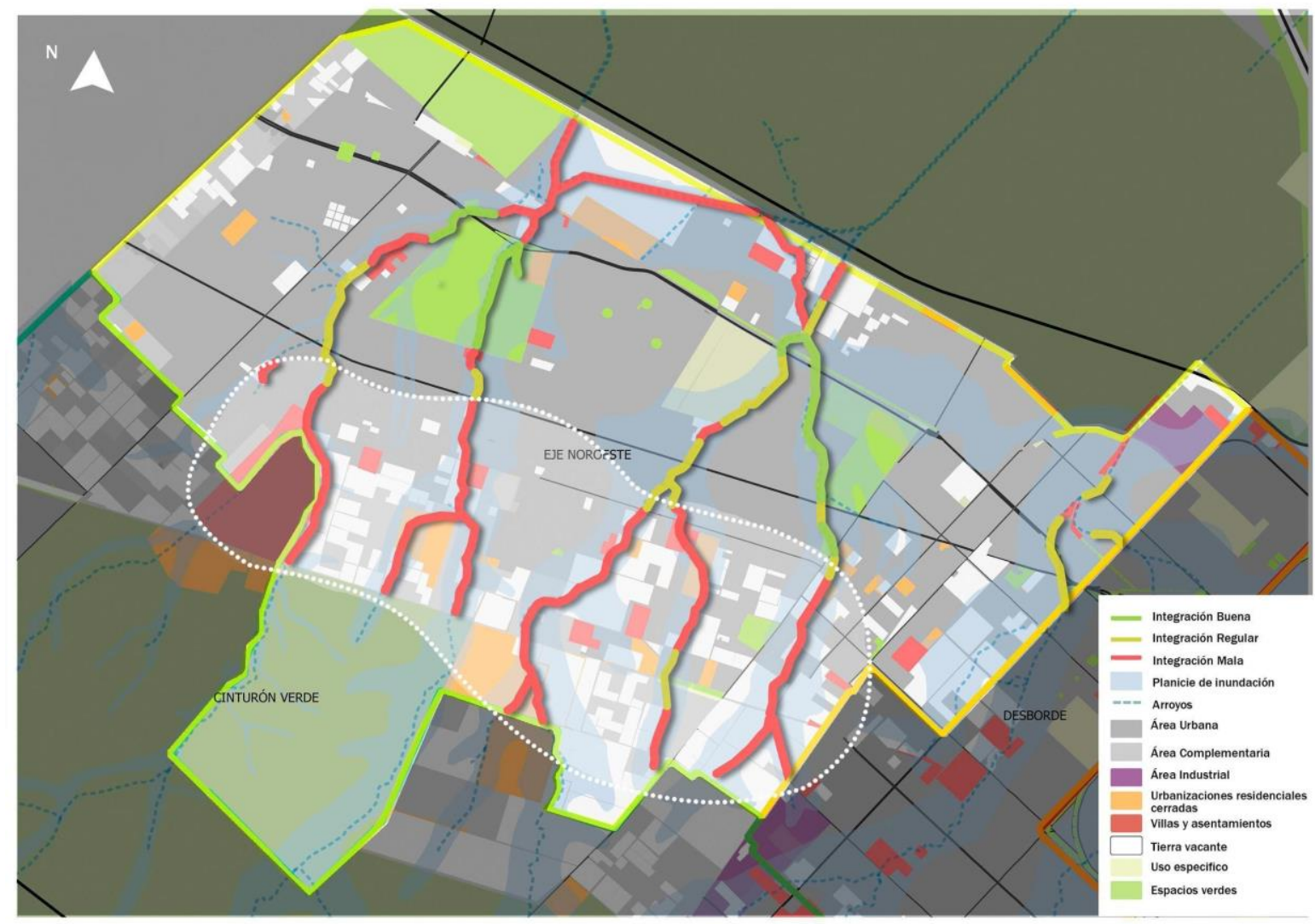

Figura 7: Eje noroeste con niveles de integración asociados a los cursos de agua. Fuente: Elaboración propia. 2017 
Se identificó una zona crítica (señalada por la línea punteada) donde se observa cómo se encuentra fragmentada la ciudad en cuanto a los usos de suelo y en esta zona es donde se concentra un nivel de integración malo. Esta zona detectada permitiría establecer áreas prioritarias de intervención de cara al futuro de la región. Como por ejemplo estrategias vinculadas a la refuncionalización de espacios en la cuenca alta de los arroyos, donde se ubican la mayoría de los barrios cerrados o estrategias de revalorización de espacios verdes existentes asociados a los cursos de agua, como es el caso de varios espacios en la cuenca media. Además de estrategias vinculadas a la refuncionalización se podrían incorporar nuevos espacios de uso público adyacentes a los cursos de agua.

También resulta interesante señalar la situación de aquellos espacios con nivel de integración medio, que se encuentran sumergidos en áreas urbanas residenciales. Estas áreas en el eje noroeste están asociadas en el imaginario a espacios con abundante vegetación y calles tranquilas, que, si bien poseen características interesantes a nivel de paisaje, no logran formular espacios de apropiación asociados a la contemplación del agua, el elemento agua es un elemento que posee gran valor en cuanto a calidad de paisaje y suele estar presente en los lugares con mayor calidad paisajística, y el paisaje. En su lugar, aparecen calles intrincadas que no logran resolver la relación agua ciudad y generan espacios de confusión para los que no conocen el área. En este sentido, respecto a la legibilidad del espacio, varios autores apuestan considerarla una variable a estudiar por métodos para determinar la calidad de diseño de un espacio urbano, sobre la base de las relaciones que se establecen entre el hombre y su medio (Ghel, 2006; Banerjee, 2001; Donadieu, 2002). Para ello coinciden en considerar los además los siguientes aspectos: permeabilidad, vitalidad o socialidad, seguridad y variabilidad. Este conjunto de cualidades sugiere la conjunción de características espaciales del tejido urbano, que ofrece un carácter abierto, variado, de fácil comprensión, con la capacidad de aceptar cambios en el tiempo.

De esta forma, y teniendo en cuenta que:

Los espacios públicos constituyen uno de los elementos para la patrimonialización afectiva de la ciudad. Se entiende aquí por patrimonialización afectiva la capacidad de generar unos vínculos afectivos estables de identificación y apego con la ciudad. Sin estos espacios públicos la ciudad pierde la capacidad de generar un patrón de actividad simbólica y significativa que favorezca la implicación de los ciudadanos en el espacio urbano y con el resto de los pobladores que lo ocupan. Y gran parte de los riesgos que hacen vulnerable los espacios urbanos derivan de la escasez y/o baja calidad de estos (Corraliza, 2009).

Si se apunta a lograr una ciudad más integrada, se debe trabajar en la constitución formal y la legibilidad de estos lugares clave, para poder establecer un carácter claro de los mismos, un carácter urbano, que no permita vacilar a la percepción de las personas que elijan quedarse a disfrutar y que constituya uno de los valores distintivos del paisaje en la ciudad.

Se decide tomar el caso del eje noroeste ya que es la zona periférica de mayor complejidad. A su vez, permite ejemplificar la situación de la periferia en su totalidad, entendiendo que el crecimiento urbano se da a partir de las principales redes viales dejando en las áreas más alejadas terrenos vacantes y nuevas urbanizaciones de baja densidad que conforman grandes áreas críticas en espacios urbanos fragmentados justo antes de encontrarse con la zona rural. Los niveles de integración determinados y medias de integración que se van a proponer a continuación son replicables en las demás zonas de la periferia platense determinadas como así también nos permiten poder aplicarlas en cualquier otra región de estudio de características similares.

\section{Medidas de integración}

Fundamentalmente para su aplicación en el área crítica, se propone determinar medidas de integración paisajísticas las cuales puedan ser incorporadas a la normativa municipal de manera de poder proteger y ordenar ciertos paisajes característicos. Estas medidas son definidas según la Guía para la elaboración de Estudios de Integración Paisajística en la Comunidad Autónoma del País Vasco del Departamento de Medioambiente, Planificación Territorial y Vivienda (2016) como: 
(L)as acciones específicas que derivan de la estrategia o estrategias de integración, que han de implementarse en el desarrollo de un proyecto $y$ que están encaminadas a evitar, reducir o corregir su impacto paisajístico, a mejorar el paisaje y la calidad del entorno o bien a compensar aquellos efectos negativos residuales.

Las medidas propuestas se establecen en base al estado de la intervención y al orden prioritario:

a) Medidas preventivas: Son aquellas medidas adoptadas en las fases de planificación y de diseño del proyecto, y en la de ejecución de la obra para evitar los impactos sobre el paisaje. Tienen que ver con la localización, el trazado, la escala, los materiales escogidos, la adaptación al relieve, entre otros. Estas medidas, deberían responder a los lineamientos planteados por un Plan de Ordenamiento y sus planes particularizados como podría ser el Plan de Forestación o el Plan de mejora en los espacios públicos.

b) Medidas correctoras: Son aquellas medidas que se incorporan al proyecto, generalmente en la fase de ejecución-construcción de la obra, para la reducción o la mitigación de la intensidad de los impactos sobre el paisaje. Tienen que ver con la visibilidad, la incorporación de barreras visuales, la alteración del relieve en el entorno, entre otros. En este punto es importante señalar el rol que juegan las empresas y el conjunto de profesionales en el rubro de la construcción y de la ingeniería civil, quienes deberán estar concientizados, capacitados y actualizados respecto de impacto paisajístico que podrían generar sus obras, entendiendo que el impacto en el paisaje conlleva consigo un impacto social y también en el ambiente. En este sentido, la conformación de barreras de hormigón sobre los canales de los arroyos que impiden las visuales al agua (entendida como mencionamos anteriormente como un elemento estructural del paisaje), limitarían las posibilidades de los habitantes de reconocer su propio paisaje. De esta forma, la construcción de recorridos paralelos a los cursos de agua y un mobiliario acorde podrían conformar proyectos que contribuyan a una mejora en la integración paisajística de la ciudad a partir de la corrección de obras que actualmente están en desarrollo. c) Medidas compensatorias: Son aquellas medidas previstas en los casos en los que no es posible la corrección ni la mitigación de los impactos sobre el paisaje y que persiguen por tanto equilibrar éstos, mediante la devolución de recompensas ambientales o sociales, luego de finalizado un proyecto. En este caso, podría considerarse, por ejemplo, la plantación de ejemplares que conformen el arbolado urbano, en otras zonas que lo requieran por fuera del caso que no es posible corregir.

Se puede decir entonces como se plantea en los Lineamientos Estratégicos para la Región Metropolitana de Buenos Aires (Subsecretaría de Urbanismo y Vivienda, 2007) que:

En la medida que los daños causados al ambiente y a la sociedad por determinadas intervenciones antrópicas no son remediados tras su abandono, desmantelamiento, o cambio de actividad, puede suceder que sus efectos tiendan a permanecer en el tiempo e incluso a constituirse en factores de degradación aún más complejos o en condicionantes para nuevos desarrollos económicos o sociales.

De esta forma, se plantea que, habiendo ya identificado los distintos niveles de integración, este tipo de medidas puedan guiar a las distintas intervenciones y proyectos realizados y a desarrollar, de manera de tender a una mejor integración paisajística principalmente en la periferia platense.

\section{Conclusiones}

Como se ha mencionado anteriormente, las zonas periféricas de la ciudad han desempeñado un rol protagónico en lo que respecta al crecimiento urbano, recibiendo aproximadamente el $70 \%$ del crecimiento demográfico desde 2010 a la fecha. Estos procesos de urbanización se hacen visibles no solo a través del aumento poblacional, sino que modifican sustancialmente la relación que mantiene la ciudad con el campo y los elementos naturales, particularmente los cursos de agua. A través de los años, se han configurado grandes zonas periurbanas que adquieren los rasgos particulares de una zona de transición y a la vez sirven de apoyo a la ciudad, pero también son susceptibles de ser absorbidas por el crecimiento urbano. La periferia platense se caracteriza 
por su dinamismo y su inmersión en los procesos de cambio morfológicos, funcionales y sociales. Esto constituye un valor distintivo del área periférica y la posiciona estratégicamente como un área de oportunidad.

Sin embargo, en relación con la integración paisajística del área periférica, se puede afirmar que el ritmo de los procesos de ocupación resulta ampliamente superior al de cualquier tipo de planificación por parte del estado. Esta situación ha derivado en una gran cantidad de espacios fragmentados, desprovistos de ciudad y de paisaje. En la periferia según el relevamiento realizado se observa que el $40 \%$ del curso de los arroyos posee un nivel de integración paisajística malo, un 35\% regular y un $25 \%$ bueno, estos datos reflejan la fragmentación del tejido como la conectividad que hay en la zona periférica. Es decir, que solo en un $25 \%$ de los casos la población puede acceder a los cursos de agua sin ningún tipo de barrera urbana, a su vez de ese $25 \%$ solo el $15 \%$ posee el equipamiento necesario para poder disfrutar de esos espacios. De esta manera, se verifica la hipótesis planteada al comienzo de la investigación de que la forma de crecimiento urbano actual juntamente con un entorno urbano fragmentado y la falta de propuestas públicas significa una pérdida en los valores de integración paisajística de los espacios públicos adyacentes a los cursos de agua.

En este sentido, aunque los emprendimientos privados cuenten con propuestas de diseño sobre estos espacios, debido a la concepción de estos como producto destinado la venta, es fundamental señalar que estos lugares estarían destinados solamente al uso de los miembros de esta urbanización cerrada, convirtiéndose entonces en un espacio de uso privado totalmente contrapuesto al concepto de ciudad, representando un $86 \%$ de la longitud catalogada con integración paisajística mala. Por otra parte, mientras que en las zonas de mayor densidad poblacional como el casco urbano los cursos de agua fueron entubados ( $y$ por lo tanto invisibilizados rompiendo su relación con el paisaje), en el área de estudio aún la mayoría de los cursos de agua permanece a cielo abierto (92\% de la longitud total), dejando libre la posibilidad de intervenir con proyectos que generen nuevos espacios públicos asociados al agua y, por lo tanto, vinculados al paisaje local.

Finalmente, creemos que mantener una visión integral de la problemática y trabajar con la amplitud conceptual necesaria resulta clave al momento de estudiar la ciudad. Es por eso que sostenemos que las nuevas formas de apropiación social y la adaptación de los espacios de esparcimiento y recreación obligan a los investigadores a reformular presupuestos y a reorientar las líneas de trabajo con una mayor conciencia respecto de la realidad urbana y generando estrategias de proyecto basadas en la compresión de las lógicas de intervención actuales más enfocadas a organizaciones por fuera del ámbito estatal o agentes privados [R] 


\section{Referencias}

Ábalos, I. (2005). Atlas Pintoresco Vol. 1: El observatorio. Barcelona: Gustavo Gili.

Arias, P. (2003). Periferias y nueva ciudad. Sevilla: Secretariado de publicaciones de la Universidad de Sevilla.

Arroyo, J. (2017). Arquitectura pública: proyectos del Estado en la poscrisis argentina de 2001. Cuaderno urbano, 22(22), 159-195. Recuperado de http://www.scielo.org.ar/scielo.php?script=sci abstra ct\&pid=S1853-36552017000100008

Banerjee, T. (2001). The Future of Public Space: Beyond Invented Streets and Reinvented Places. Journal of the American Planning Association, 67(1), 9-24. https://doi.org/10.1080/01944360108976352

Birche, M. y Jensen, K. (2016). Hacia el desarrollo urbano de la región la plata. Una nueva perspectiva para el abordaje de la complejidad territorial en IFoU - The 9th International Conference of the International Forum on Urbanism.

Borja, J. (2003). La ciudad conquistada. Madrid: Alianza Editorial.

Celemín, J. P., Mikkelsen, C. A. y Velázquez, G. A. (2015). La calidad de vida desde una perspectiva geográfica: integración de indicadores objetivos y subjetivos. $R e$ vista Universitaria de Geografía, 24(1), 63-84. Recuperado de: http://bibliotecadigital.uns.edu.ar/scielo.p hp?script=sci arttext\&pid=S1852-426520150011000 04\&lng=es\&nrm=iso

Corraliza, J. A. (2009). Emoción y espacios públicos: La experiencia humana de los escenarios urbanos. Madrid: Universidad Autónoma de Madrid.

Clichevsky, N. (2001). Tres casos de utilización de tierra vacante en el Área Metropolitana de Buenos Aires. Cambridge: Lincoln Institute of Land Policy.

Dematteis, G. (1998). Suburbanización y periurbanización. Ciudades anglosajonas y ciudades latinas. En F.J. Monclús (Ed.) La ciudad dispersa (pp. 1-4). Barcelona: Centro de Cultura Contemporánea de Barcelona.

Departamento de Medioambiente, Planificación Territorial y Vivienda del Gobierno Vasco (2016). Guía para la elaboración de Estudios de Integración Paisajística en la Comunidad Autónoma del País Vasco. Recuperado de: http://www.euskadi.eus/contenidos/ informacion/presentacion paisaia/es def/adjuntos/G uia\%20paisaje FINAL.pdf
Real Academia Española (2017). Integrar. En Diccionario de la lengua española (23.a ed.). Recuperado de http://dle.rae.es/?id=LqKFoJI

Donadieu, P. (2002). La sociedad paisajista. La Plata: Editorial de la Universidad Nacional de La Plata.

García, M. (2015). Perspectivas paisajísticas para la planificación. Constelaciones, 3, 125-139. Recuperado de https://revistaconstelaciones.wordpress.com/20 15/05/15/perspectivas-paisajisticas-para-la-planific acion/

Ghel, J. (2006). La humanización del espacio, Barcelona: Editorial Reverte

Herrera, L. y Pecht, W. (1976). Crecimiento urbano de América latina centro latinoamericano de demografía. CELADE: Santiago de Chile. Recuperado de https://repositorio.cepal.org/bitstream/handle/1136 2/7415/S301361H565V1 es.pdf?sequence=1

Hiernaux, D. y Lindón, A. (2004). La periferia: voz y sentido en los estudios urbanos. Papeles de Población, 10(42), 101-123. Recuperado de http://www.scielo.org.mx/s cielo.php?script=sci arttext\&pid=S1405-7425200400 0400005\&lng=es\&nrm=iso

Jensen, K. y Birche, M. (2017 a). Espacio verde: elemento clave en la gestión urbana. Revista Ciudades, 115.

Jensen, K. y Birche, M. (2017 b). Vulnerabilidad al cambio climático: las inundaciones en la cuenca del arroyo El gato. Revista CIS, 14(23), 77-100. Recuperado de http://revistacis.techo.org/index.php/Journal/article/ view/12

Lanfranchi, G., Duarte, J. I. y Granero, G. (2018). Documento de políticas públicas. Resumen ejecutivo. Centro de Implementación de Políticas Públicas para la Equidad y el Crecimiento.

Municipalidad de La Plata. (2004). Código de Espacio Público de La Plata. Recuperado de http://www.con cejodeliberante.laplata.gov.ar/digesto/cod espacio/o r9880 indice.asp

ONU Habitat (2012). Estado de las ciudades de América Latina y el Caribe. Rumbo a una nueva transición urbana. Brasil: ONU Habitat.

Pascual, A. y Peña, J. (2012). Espacios abiertos de uso público. Arquitectura y Urbanismo, 33(1), 25-42. Recuperado de http://scielo.sld.cu/scielo.php?scr ipt=sci arttext\&pid=S1815-58982012000100003 
Romanazzi, P. y Urbiztondo, A. (2011). Estudios Hidrológicos - Hidráulicos- Ambientales en la cuenca del Arroyo del Gato. Recuperado de https://www.ing. unlp.edu.ar/sitio/investigacion/archivos/jornadas201 1/ih07.pdf

Rueda, S. (1997). La ciudad compacta y diversa frente a la conurbación difusa. Recuperado de: http://habitat.aq.upm.es/cs/p2/a009.html

Salcedo, R. (2002). El espacio público en el debate actual: Una reflexión crítica sobre el urbanismo postmoderno. EURE (Santiago), 28(84), 5-19. https://doi.org/10.4067/s0250-71612002008400001

Subsecretaría de Urbanismo y Vivienda (2007). Lineamientos Estratégicos para la Región Metropolitana de Buenos Aires. Recuperado de: https://www.mininterior.gov.ar/planificacion/pdf/pla nes-reg/Lineamientos-Estrategicos-para-la-Region-

Metropolitana-de-Buenos-Aires.pdf
Silva, R., y Fernández, V. (2015). Los paisajes culturales de Unesco desde la perspectiva de América Latina y el Caribe. Conceptualizaciones, situaciones y potencialidades. Revista INVI, 30(85), 181-212. https://doi.org/10.4067/s0718-83582015000300006

Rotger, D. (2017). Paisaje fluvial y planificación del territorio. Perspectivas de integración en la Región Metropolitana de Buenos Aires. Revista de Urbanismo, 37, .1-15. http://dx.doi.org/10.5354/ru.v0i37.47085

Sanabria, T. H. y Ramirez, J. F. (2017). Ciudad compacta vs. ciudad difusa. Ecos antiguos y recientes para las políticas de planeación territorial y espacial. Cuaderno Urbano, 22(22), 29-52. Recuperado de http://www. scielo.org.ar/scielo.php?script=sci abstract\&pid=\$185 3-36552017000100002 